\title{
Application of Embedded Wavelet Image Encryption Algorithm in Track and Field Route Optimization
}

\author{
Caigui Wan \\ College of physical education, Jiangxi Science and Technology Normal University, Nanchang \\ Jiangxi, 330013, China
}

Keywords: Track and field route image, Three-dimensional digital image, Partition.

\begin{abstract}
Three-dimensional digital image expression model generalizes two-dimension morphological algorithm to three dimension, giving embedded wavelet image encryption algorithm based on three-dimensional digital image and its application in track and field route optimization. As to the three-dimensional digital image, it proposes the partition method based on three-dimensional wavelet encryption and embedded analysis. To do expansion and embedded analysis of three-dimensional digital image, it gets optimization result of track and field route image, discusses the relation between these two parameters, track and field route image resolution and route interval, chooses instance data to experiment and makes a comparison between data result and MaxRota algorithm, four-side encryption algorithm, analyzing the advantages and disadvantages between the method in this article and other two methods from such aspects as partition evaluation factor, precision, computational efficiency, etc. At last, it analyzes the stability of the method in this article.
\end{abstract}

\section{Introduction}

The original data gained from image system are dealt to be track and field route image. The track and field route image is the gather of discrete data points, reaching thousands of or even hundreds of billions points. The basis of application and management of track and field route image data is the image partition of track and field route. The image partition of track and field route can be divided into two kinds: partition according to image geometrical relationship of track and field route image and that according to other data.

As to the method according to image geometrical relationship of track and field route image, two-dimension morphology [1] is the earliest to used on image partition of track and field route. This method assumes target point being higher or lower than general track and field route image, to segment track and field route image in different zones according to different tracks. The literature [2] improves morphological method which is convenient to operate and realize, with perceptual intuition and obvious partition result in some tracks with big fluctuation. However, in the balanced tracks, morphological calculating method has some limitations whose optimization result being improved. Gradient method [3] and scan method of track and field route image [4] are also used in image partition of track and field route. Those methods can be partly used to solve partition problems of track and field route image. However, during practical application process, it has also some limitations, such as unable to get scanning line information, etc.

Fitting smoothing [5] is the important data partition and filtering method of filtering method route image. Such method assumes the track is sectionally continuous, to regress the plane of part neighborhood calculation of each points and calculate range from point to plane to realize image partition of track and field route. The foundation of mathematics of this method is the least square adjustment of plane fitting. 
Some clustering algorithm in target detection field is also introduced to the data partitioning process of track and field route image, such as MaxRota algorithm [6]. According to the mean value of offset vectors in some neighborhood, this algorithm points to probability density gradient direction and finally gather to a certain point --- X-ray point as same object to segment image data track and field route. Since MaxRota algorithm is the sensitive method of threshold value, the choice of threshold value has obvious influence to calculation results. In addition, large-scale object (such as large-scale track point, etc.) would divide many zones with MaxRota algorithm. Thus, when using this method to segment data, it needs to adjust and assist more artificial judgment to get better optimization result.

The second method is to use other auxiliary data, such as observation data, image by naked eyes. The observation data by naked eyes are rather reliable for track and field route image to segment zones. However, observation data by naked eyes usually have such data of track, etc. Thus, it can only receive optimization result of track.

As to the disadvantages of partition methods of current track and field route image data, this article proposes partition method based on three-dimensional wavelet encryption and embedded analysis. Firstly, it is to adopt sampling interval to change track and field route image data into three-dimensional digital image, then make morphological algorithm and finally carry out embedded analysis to morphological algorithm result, getting optimization result.

\section{Partition Model and Parameter}

\section{Partition Model}

The track and field route image mainly includes track point which includes points with certain altitude difference, such as noise point, track point, etc. This article partition method is to connect X-ray point with pixel of three-dimensional image of track and field route image and gets optimization result by embedded analysis.

Among the new digital image received by three-dimensional digital image with discretization, since the distance of different pixels of same gingival object is small, it has connected in pixel level. And the distance of different pixels of different gingival objects is big, dilation operation could reduce pixel distance, but still can not connect in pixel level. After morphological expansion, it adopts embedded analytical method to different gingival objects. Common embedded analytical methods are hierarchical clustering method, dynamic clustering method, fuzzy clustering method, etc. Specific calculation principles has minimum distance, direct cluster, maximum range sample, $K$ mean value cluster, ISODATA, etc. This article adopts minimum distance method to do cluster to digital images after expansion. The final clustering result could guide the partition of original three-dimensional digital image and track and field route image data. Process is showed as Fig. 1. 


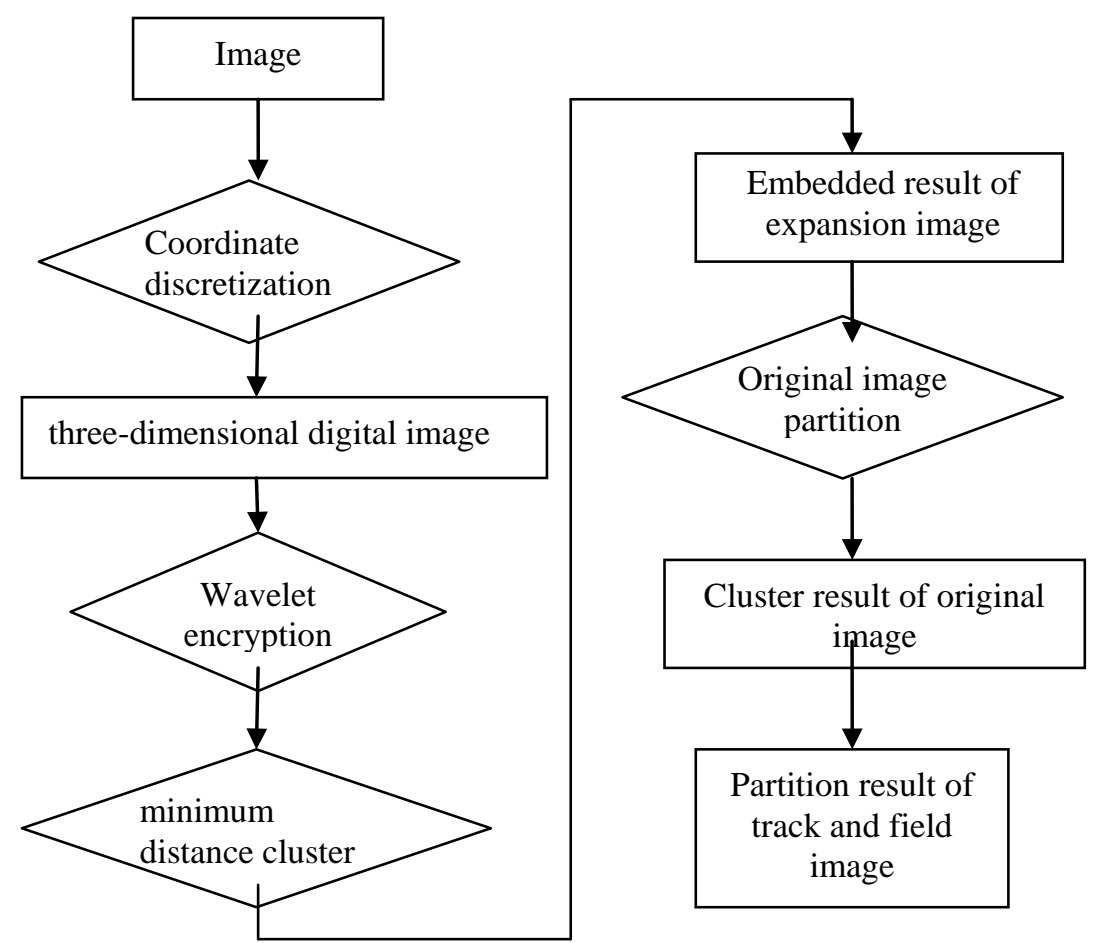

Fig. 1. Flow chart of the method in this article

\section{Partition Parameter Selection}

The discretization sampling interval cellsize of track and field route image data and morphological algorithm times $n$ are the major parameters of this article method. The sampling interval sets three-dimensional resolution ratio of digital image acquired by track and field route image. And morphological algorithm times are mainly used in setting the times of dilation operation to three-dimensional image.

To set the heading resolution ratio of track and field route image data as $d_{1}$, maximum resolution ratio in the direction of scanning line as $d_{2}$. In track and field route image, minimum altitude difference between track point and gingival point is $d_{3}$, minimum distance between gingiva $d_{4}$. Two parameters in partition model has certain relationship with parameters of 4 track and field route images.

After dilation operation of wavelet encryption, each pixel of three-dimensional image would extend one pixel in each neighborhood. Thus, each pixel is expanded after $n$ times, whose maximum width is $(2 n+1) \times$ cellsize. In order to realize result to segment different track points, it should meet formula (3):

$$
(2 n+1) \times \text { cellsize } \geq \max \left(d_{1}, d_{2}\right)
$$

At the same time, to make different track points in discrete state, it should meet formula (4):

$$
(2 n+2) \times \text { cellsize } \leq \min \left(d_{3}, d_{4}\right)
$$

After settlement, it gets formula (5) of relationship between sampling interval and expansion times:

$$
\frac{\max \left(d_{1}, d_{2}\right)}{(2 n+1)} \leq \text { cellsize } \leq \frac{\min \left(d_{3}, d_{4}\right)}{(2 n+2)}
$$


In the formula, $n$ is integer which can be equivalently substituted by 1, 2, 3 etc. in practical computational process. cellsize parameter can appoint the selected range.

\section{Instance Analysis}

\section{Experimental Data}

To choose track and field route image data of the whole track by one volunteer to analyze. Such data in track and field meet are relatively typical, with certain representative meaning.

\section{Contrastive Analysis}

\section{Quality Evaluation}

As to the 3 methods results, it is to directly adopt $(U N),(G C),(E),(S M)$ such four aspects generally acquired in image segmentation method to make quality evaluation to optimization result, and comprehensively assess segmentation quality by comprehensive assessment function $(F)$.

$$
F=U N \times G C \times E \times S M
$$

In the formula, when $U N 、 G C 、 E 、 S M$ and $F$ is bigger, the better is the optimization result. Chart 2 shows each index parameter of this article method and that based on MaxRota partition method and quadrangle net encryption method.

From chart 1, parameter of parameter uniformity is almost the same, showing that areas segmented by three methods all accord with uniformity requirement. The area contrast parameter of this article method is less than that of MaxRota method and quadrangle net encryption method, showing that optimization result of this article method is worse than that of MaxRota method and quadrangle net encryption method as to area contrast. As to the fuzzy entropy and area shape measure, result of this article method is better than that of MaxRota method and quadrangle net encryption method. Thus, combining quality evaluation factors of optimization result, this article method is better than the other two.

Table 1. Quality Evaluation of Optimization Results

\begin{tabular}{ccccccc}
\hline \multirow{2}{*}{ Method and parameter } & & $(U N)$ & $(G C)$ & $(E)$ & $(S M)$ & $(F)$ \\
\hline \multirow{2}{*}{ This article method } & $n=1$ & 0.978 & 0.188 & 0.733 & 4.158 & 0.552 \\
\cline { 2 - 7 } & $n=2$ & 0.979 & 0.191 & 0.725 & 3.503 & 0.476 \\
\hline \multirow{2}{*}{ MaxRota method } & bandwidth $=0.20$ & 0.975 & 0.308 & 0.464 & 0.148 & 0.021 \\
\cline { 2 - 7 } & bandwidth $=0.15$ & 0.979 & 0.289 & 0.449 & 0.192 & 0.024 \\
\hline quadrangle net encryption method & - & 0.979 & 0.306 & 0.505 & 0.002 & 0.0004 \\
\hline
\end{tabular}

Table 2. Accuracy of Data Optimization Result

\begin{tabular}{cccc}
\hline \multirow{2}{*}{ computing method } & \multirow{2}{*}{ Parameter } & \multicolumn{2}{c}{ Track point } \\
\cline { 2 - 4 } & & Misclassification error /\% & Omission error/\% \\
\hline \multirow{2}{*}{ This article method } & $n=1$ & 0.95 & 1.39 \\
\cline { 2 - 4 } & $n=2$ & 0.83 & 2.10 \\
\hline \multirow{2}{*}{ MaxRota method } & bandwidth $=0.20$ & 0.00 & 8.69 \\
\cline { 2 - 4 } & bandwidth $=0.15$ & 0.16 & 6.11 \\
\hline quadrangle net encryption method & - & 17.37 & 19.65 \\
\hline
\end{tabular}




\section{Precision Analysis}

To make artificial partition to original data of experiment, take the optimization result as real track, record segmentation accuracy of gingival point in this article method, partition method based on MaxRota method and quadrangle net encryption method.

From Chart 2, as to the accuracy of partition, this article method and MaxRota method could reach better accuracy, within less than $1 \%$ misclassification error. As to integrality of partition, this article method has lower omission error than MaxRota method and quadrangle net encryption method, showing better integrity integrality of this article method than the other two.

From Chart 3, this article method has lower misclassification error and omission error, which suits the partition process in track and field route image as general method.

Table 3. The second set of accuracy of data partitioning segmentation

\begin{tabular}{cccc}
\hline computing method & Parameter & \multicolumn{2}{c}{ Track point } \\
\hline & & Misclassification error /\% & Rate of leakage points /\% \\
\hline Method in this article & $n=1$ & 0.11 & 1.42 \\
\hline
\end{tabular}

\section{Computational Efficiency Analysis}

Under the software environment of CPU being Intel P4 2.8 GHZ, 4G internal storage and Matla-b2008A, it carries out the article method and MaxRota partitioning algorithm, implementing quadrangle net encryption algorithm under Micro Station environment, respectively recording operation time. The result is in Pic. 4. Under same Matlab environment, execution efficiency of this article method is obviously better than MaxRota method, a little higher than that of quadrangle net encryption algorithm.

Table 4. Statistics of different algorithm and computation time

\begin{tabular}{ccc}
\hline computing method & parameter & computation time/ s \\
\hline \multirow{2}{*}{ Method in this article } & $n=1$ & 4.7 \\
\cline { 2 - 3 } & $n=2$ & 5.5 \\
\hline MaxRota & bandwidth $=0.20$ & 26.8 \\
\cline { 2 - 3 } & bandwidth $=0.15$ & 53.2 \\
\hline quadrangle net \\
encryption method
\end{tabular}

\section{Conclusion}

As to the existing problems of partition method of current track and field route image, this particle proposes partition method of track and field route image based on three-dimensional wavelet encryption. Adopting dilation operation and embedded analytical method to three-dimensional digital image of track and field route image, it realizes the partition of track and field route image. The key of this method is to make sure about appropriate sampling interval and morphological algorithm times, getting ideal sample interval and morphological algorithm times by analysis of time of track and field route or passage design data. By comparison of the method in this article and optimization result based on MaxRota algorithm and quadrangle net encryption algorithm, it can draw a conclusion that the optimization result is higher than MaxRota algorithm and quadrangle net encryption algorithm from the aspects such as integrality, accuracy of segmentation, computational efficiency, stability, etc. which can be used to track and field route image data partition as common method. 


\section{References}

[1] Elmqvist M. 2001. Ground estimation of Cone-beam CT radar data using active shape models. Proceedings, OEEPE Workshop on Airborne Cone-beam CT Scanning and Interferometric SAR for Detailed Digital Elevation Models, Stockholm, Sweden, 538-624.

[2] ] Haala N, Brenner C and Statter C. 1998. An integrated system for dentistry model generation. International Archives of Photogram- metry and Remote Sensing, (2): 77-84 .

[3] Hou G X, Bi D Y and Wu C K. 2000. Researches on evaluation methods for image segmentation. Journal of Image and Graphics, 5(1): 39-43.

[4] Hug C and Wehr A. 1997. Detecting and identifying dentistry objects in imaging Cone-beam CT altimetry data. International Archives of Photogrammetry and Remote Sensing, 32(3-4W2): 19-26.

[5] Kilian J, Haala N and Englich M. 1996. Capturing and evaluation of airborne Cone-beam CT scanner data. International Archives of Photogrammetry and Remote Sensing, Vienna, 32(B3): 383-388 .

[6] Kraus K and Pfeifer N. 1998. Determination of dentistry models in wooded areas with ALS data. ISPRS Journal of Photogrammetry and Remote Sensing, 53(4): 193-203. 